PharmacoEconomics \& Outcomes News 879, p28 - 29 May 2021

\title{
Tocilizumab cost effective in reducing COVID-19-related deaths
}

Treatment with tocilizumab in combination with dexamethasone appears to be cost effective in reducing COVID-19-related deaths, according to findings of a US study published in Clinical Infectious Diseases.

A decision-tree model populated with data from the randomised RECOVERY trial, which was conducted in the UK, was used to evaluate the cost effectiveness of tocilizumab plus dexamethasone, compared with dexamethasone alone or best supportive care (BSC) alone, in patients with severe COVID-19 infection. Cost effectiveness was assessed from a US perspective. Assumed drug costs were \$5304 for tocilizumab and \$12 for dexamethasone, and assumed annual healthcare costs in COVID-19 survivors were $\$ 6929$ per year.

Tocilizumab plus dexamethasone, dexamethasone alone and BSC alone were estimated to achieve a gain 9.36, 8.66 and 8.43 QALYs, respectively, at a total cost of \$83 130, \$71 630 and \$69 700, respectively. The estimated incremental cost-effectiveness ratio (ICER) for combination therapy versus dexamethasone alone was \$16 520 per QALY gained in the base-case scenario, and \$26 840 per QALY gained in a scenario with a lower mortality rate (14.6\%) based on data from New York City.

In probability sensitivity analysis, the cost-effectiveness acceptability curve showed that tocilizumab plus dexamethasone had over $98 \%$ and $76 \%$ probability of being cost effective in the base-case scenario and lower mortality scenario, respectively, at a willingness-to-pay threshold of \$100 000 per QALY gained.

"Tocilizumab in addition to dexamethasone is a cost effective intervention for individuals severely ill with COVID-19," concluded the authors.

Sinha P, et al. Combination therapy with tocilizumab and dexamethasone cost-effectively reduces Coronavirus disease 2019 mortality. Clinical Infectious Diseases : 6 May 2021. Available from: URL: http://doi.org/10.1093/cid/ciab409 